\title{
SELF-ADJOINT TOEPLITZ OPERATORS AND ASSOCIATED ORTHONORMAL FUNCTIONS ${ }^{1}$
}

\author{
MARVIN ROSENBLUM
}

1. Introduction. Let $l^{2}$ be the Hilbert space of square summable sequences $f=\left(f_{0}, f_{1}, f_{2}, \cdots\right) . l^{2}$ is isomorphic to the space $H^{2}$ of functions holomorphic in the unit disk $\Delta$ with square integrable boundary values, under the map

$$
f \rightarrow F, \quad F(z)=\sum_{n=0}^{\infty} f_{n} z^{n} .
$$

A Toeplitz operator is an $l^{2}$ linear operator $T$ to which corresponds a function $W$ on the unit circle $\Gamma$, such that under the isomorphism (1.1) we have for the inner product

$$
\langle T f, g\rangle=\frac{1}{2 \pi} \int_{-\pi}^{\pi} W(\theta) F\left(e^{i \theta}\right) G^{*}\left(e^{i \theta}\right) d \theta .
$$

Here * denotes complex conjugation.

This work is concerned with the concrete spectral theory of Toeplitz operators that are associated with functions $W$ that satisfy the following two hypotheses:

(i) $W$ is real, bounded below, and absolutely integrable on $\Gamma$, but it is not equivalent to a constant function.

(ii) For each real $\lambda$ the set $\Gamma_{\lambda}=\{\theta: W(\theta) \leqq \lambda\}$ is, modulo a set of measure zero, an arc of the circle.

By concrete spectral theory we mean ${ }^{2}$ that we exhibit an explicit sigma-finite measure $\rho$ on $(-\infty, \infty)$ and an explicit unitary correspondence $U: l^{2} \rightarrow L^{2}(d \rho)$ such that $U T U^{-1}=M$, where $M$ is the multiplication operator on $L^{2}(d \rho)$ which sends $g(\lambda)$ into $\lambda g(\lambda)$. Hypothesis (i) implies that $T$ is bounded below, so its Friedrichs extension (again named $T$ ) is self-adjoint.

I am deeply indebted to the referee, who simplified the proof and helped clarify the exposition.

The results obtained are conveniently described in terms of the set of vectors $k(u) \in l^{2}$ defined for each $u \in \Delta$ by

Received by the editors May 12, 1960 and, in revised form, June 29, 1961.

1 The author is a National Science Foundation fellow.

2 This description applies only to operators with simple spectrum. The hypotheses given guarantee that $T$ has simple spectrum, but this is not in general true for Toeplitz operators, cf. [6]. 


$$
k_{n}(u)=u^{n}, \quad k_{0}(0)=1 .
$$

Under the correspondence $(1.1)$ it follows that $K(u ; z)=(1-u z)^{-1}$, and thus for each $f \in l^{2}$ we have

$$
\left\langle f, k\left(u^{*}\right)\right\rangle=F(u) .
$$

Let $E$ be the spectral measure of $T$. In Theorem 3 we find that there is an absolutely continuous measure $\rho$ on the line given by (3.3) and a collection of functions $\Phi(u ; \lambda)$ given by (3.2) such that for all $u, v \in \Delta$ and each real Borel set $\Lambda$

$$
\langle E(\Lambda) k(u), k(v)\rangle=\int_{\Delta} \Phi(u ; \lambda) \Phi^{*}(v, \lambda) d \rho(\lambda) .
$$

According to (2.7) and Lemma 1 the functions $\Phi(u ; \lambda)$ are for almost all $\lambda$ holomorphic functions of $u \in \Delta$ with the Maclaurin expansion

$$
\Phi(u ; \lambda)=\sum_{n=0}^{\infty} \phi_{n}(\lambda) u^{n} .
$$

For fixed $u \in \Delta, \Phi(u ; \lambda) \in L^{2}(d \rho)$. Now the transformation $k(u)$ $\rightarrow \Phi(u ; \lambda)$ defined on the set $\mathcal{K}=\{k(u): u \in \Delta\}$ in $l^{2}$ with range in $L^{2}(d \rho)$ preserves inner products as can be seen by taking $\Lambda=(-\infty, \infty)$ in (1.5). Since $\mathcal{K}$ is total in $l^{2}$, cf. (1.4), it follows that there exists a unique isometry $U: l^{2} \rightarrow L^{2}(d \rho)$ such that $U k(u)=\Phi(u ; \lambda)$. This transformation has the explicit form below obtained from (1.3) and (1.6)?

$$
U f=\sum_{0}^{\infty} f_{n} \phi_{n} .
$$

We next note that (1.5) implies that for each real Borel set $\Lambda, U$ sends $E(\Lambda) k(0)$ into the product of the indicator function of $\Lambda$ and $\Phi(0 ; \lambda)$. Since by $(3.2) \Phi(0 ; \lambda)$ is almost everywhere nonzero, it follows that the range of $U$ is $L^{2}(d \rho)$. Thus $U$ is a unitary mapping of $l^{2}$ onto $L^{2}(d \rho)$.

As a corollary of the fact that (1.7) is a unitary equivalence we conclude that $\left\{\phi_{n}\right\}$ is a complete orthonormal set in $L^{2}(d \rho)$. So we see that $\Phi(u ; \lambda)$ is a generating function for a complete orthonormal set of functions in $L^{2}(d \rho)$. In the examples at the end we specify $W$ so as to obtain certain Gegenbauer and Pollaczek polynomials.

2. Analysis of Toeplitz matrices. Suppose now that hypothesis (i) is satisfied, and set $\lambda_{0}=$ ess inf $W$. It is known, [1], that whenever $\lambda<\lambda_{0}$ there is a factorization

$$
W(\theta)-\lambda=\left|H_{\lambda}\left(e^{i \theta}\right)\right|^{2},
$$


where $H_{\lambda}$ is an outer function in $H^{2}$, i.e., the set $\left\{z^{n} H_{\lambda}(z)\right\}_{n=0}^{\infty}$ is total in $H^{2} . H_{\lambda}$ is uniquely specified if we impose the normalization $H_{\lambda}(0)$ $>0$. The explicit formula is

$$
H_{\lambda}(u)=\exp \int_{-\pi}^{\pi} \log (W(\theta)-\lambda) P^{*}\left(u^{*}, \theta\right) d \theta, \quad u \in \Delta
$$

where

$$
P(u, \theta)=\frac{1}{4 \pi}\left(1+u e^{i \theta}\right)\left(1-u e^{i \theta}\right)^{-1} .
$$

Thus whenever $\lambda<\lambda_{0}$ and $T$ is given by (1.2) we may write $T-\lambda=S_{\lambda}^{*} S_{\lambda}$, where $S_{\lambda}$ is the operator which under the map (1.1) transforms into multiplication by $H_{\lambda}$, i.e.

$$
S_{\lambda} f \rightarrow B_{\lambda} F \text {. }
$$

From (1.4) we easily compute that

$$
S_{\lambda}^{*} k(u)=H_{\lambda}^{*}\left(u^{*}\right) k(u) \text {. }
$$

Since $H_{\lambda}$ is an outer function, $S_{\lambda}$ and $S_{\lambda}^{*}$ have densely defined inverses. Hence $(T-\lambda)^{-1}=S_{\lambda}^{-1} S_{\lambda}^{*-1}$. Using (2.4) we see that

$$
\left\langle(T-\lambda)^{-1} k(u), k(v)\right\rangle=H_{\lambda}^{*-1}\left(u^{*}\right) H_{\lambda}^{-1}\left(v^{*}\right)\langle k(u), k(v)\rangle .
$$

It is useful to rewrite this formula using (2.2) and the fact that $\langle k(u), k(v)\rangle=\left(1-u v^{*}\right)^{-1}$. We obtain

$$
\begin{aligned}
& \left\langle(T-\lambda)^{-1} k(u), k(v)\right\rangle \\
& =\left(1-u v^{*}\right)^{-1} \exp -\int_{-\pi}^{\pi} \log (W(\theta)-\lambda)\left[P(u, \theta)+P^{*}(v, \theta)\right] d \theta .
\end{aligned}
$$

For $u, v \in \Delta$, the right hand side of $\left(2.5^{\prime}\right)$ is holomorphic in the $\lambda$ plane cut along the real axis from $\lambda_{0}$ to $\infty$. Thus $\left(2.5^{\prime}\right)$ provides an analytic continuation of the resolvent of $T$. We will apply the Stieltjes inversion formula

$$
\begin{aligned}
d\langle E(\lambda) k(u), k(v)\rangle / d \lambda=\frac{1}{2 \pi i} \lim _{\epsilon \downarrow}\{ & \left\langle(T-\lambda-i \epsilon)^{-1} k(u), k(v)\right\rangle \\
& \left.-\left\langle(T-\lambda+i \epsilon)^{-1} k(u), k(v)\right\rangle\right\}
\end{aligned}
$$

to $\left(2.5^{\prime}\right)$, making use of the following

LEMma 1. For almost all real $\lambda$

$$
\int_{-\pi}^{\pi} \log |W(\theta)-\lambda| d \theta>-\infty \text {. }
$$


Proof. Let $z=\lambda+i \epsilon, \epsilon>0$, and consider $I(z)=\int_{-\pi}^{\pi} \log (W(\theta)-z) d \theta$. This equals

$$
k \int_{-\pi}^{\pi} \log \left[(W(\theta)-z)\left(1+W(\theta)^{2}\right)^{-1 / 2}\right] d \theta
$$

where $k=(1 / 2) \int_{-\pi}^{\pi} \log \left[1+W(\theta)^{2}\right] d \theta$. Thus

$$
I(z)=\int_{-\infty}^{\infty} \log \left[(t-z)\left(1+t^{2}\right)^{-1 / 2}\right] d \alpha(t),
$$

where $\alpha$ is bounded and monotone. Upon partial integration we have $I(z)=\int_{-\infty}^{\infty}\left[(t-z)^{-1}-t\left(1+t^{2}\right)^{-1}\right] \alpha(t) d t$. Thus $\lim _{\epsilon \downarrow 0} I(z)$ exists a.e. and $\lim _{\epsilon \downarrow 0} \operatorname{Re} I(z)=\lim _{\epsilon \downarrow 0} \int_{-\pi}^{\pi} \log |W(\theta)-\lambda+i \epsilon| d \theta$ is finite a.e. The lemma now follows from this by monotone convergence.

The lemma allows us to conclude that

$$
\Psi(u ; \lambda)=\exp -\int_{-\pi}^{\pi} \log |W(\theta)-\lambda| P(u, \theta) d \theta
$$

defines for almost all $\lambda$ a holomorphic function of $u \in \Delta$. Let $\Gamma_{\lambda}$ $=\{\theta: W(\theta) \leqq \lambda\}$. Evidently

$$
\begin{aligned}
\lim _{\epsilon \downarrow 0} \log (W(\theta)-\lambda \pm i \epsilon) & =\log |W(\theta)-\lambda| \text { when } \theta \notin \Gamma_{\lambda} \\
& =\log |W(\theta)-\lambda| \pm \pi i \text { when } \theta \in \Gamma_{\lambda} .
\end{aligned}
$$

We can now calculate the right-hand side of $(2.6)$ from $\left(2.5^{\prime}\right)$. The result is

THEOREM 1. Let $T$ be a Toeplitz operator defined by (1.2), where $W$ satisfies hypothesis (i). Then the resolution of the identity $E(\lambda)$ of $T$ satisfies a.e.

$$
\begin{aligned}
& d\langle E(\lambda) k(u), k(v)\rangle / d \lambda \\
& \quad=\pi^{-1} \Psi(u ; \lambda) \Psi^{*}(v ; \lambda)\left(1-u v^{*}\right)^{-1} \sin \left\{\pi \int_{\Gamma_{\lambda}}\left[P(u, \theta)+P^{*}(v, \theta)\right] d \theta\right\} .
\end{aligned}
$$

Theorem 1 describes the absolutely continuous part of $T$. That this is a complete description follows from

THEOREM 2. Let $T$ be a Toeplitz operator defined by (1.2) where $W$ satisfies hypothesis (i). Then the spectral resolution of $T$ is weakly absolutely continuous with respect to Lebesgue measure.

Proof. See [6]. Theorem 2 makes use of the assumption that $W$ is not equivalent to a constant. The two theorems combined show that the spectrum of $T$ is purely continuous and consists of the closed 
interval [ess inf $W$, ess sup $W$ ]. This result is known, cf. [3 and 4]. What remains to be done is to exploit Theorem 1 . This will require the use of hypothesis (ii).

3. Concrete spectral theory. We shall need the simple computational

LEMMA 2. If $0 \leqq b-a \leqq 2 \pi$,

$$
\int_{a}^{b} P(u, \theta) d \theta=\frac{1}{4 \pi}(b-a)+\frac{1}{2 \pi i} \log \left[\left(1-u e^{i a}\right)\left(1-u e^{i b}\right)^{-1}\right] .
$$

Hypothesis (i) says that $\Gamma_{\lambda}=\{a(\lambda) \leqq \theta \leqq b(\lambda)\}$ where $0 \leqq b(\lambda)-a(\lambda)$ $\leqq 2 \pi$. From Lemma 2 we have

$$
\begin{aligned}
& \pi \int_{\Gamma_{\lambda}}\left[P(u, \theta)+P^{*}(v, \theta)\right] d \theta \\
& =\frac{1}{2}(b-a)+(2 i)^{-1} \log \left[\left(1-u e^{i a}\right)\left(1-v^{*} e^{-i b}\right)\left(1-u e^{i b}\right)^{-1}\left(1-v^{*} e^{-i a}\right)^{-1}\right]
\end{aligned}
$$

where $a=a(\lambda)$ and $b=b(\lambda)$. The main theorem is

Theorem 3. Let $T$ be a Toeplitz operator defined by (1.2) where $W$ satisfies hypotheses (i) and (ii). Then in the spectral decomposition of $T$ we have for each real Borel set $\Lambda$

$$
\langle E(\Lambda) k(u), k(v)\rangle=\int_{\Lambda} \Phi(u ; \lambda) \Phi^{*}(v ; \lambda) d \rho(\lambda)
$$

where

$$
\Phi(u ; \lambda)=\Psi(u ; \lambda)\left(1-u e^{i a(\lambda)}\right)^{-1 / 2}\left(1-u e^{i b(\lambda)}\right)^{-1 / 2}
$$

and

$$
d \rho(\lambda)=\pi^{-1} \sin \frac{1}{2}[b(\lambda)-a(\lambda)] d \lambda
$$

Proof. With the aid of (3.1) the sine term in the formula of Theorem 1 can be calculated. We obtain

$$
d\langle E(\lambda) k(u), k(v)\rangle / d \lambda=\Phi(u ; \lambda) \Phi^{*}(v, \lambda) \rho^{\prime}(\lambda)
$$

for almost all $\lambda$ where $\rho^{\prime}(\lambda)=d \rho(\lambda) / d \lambda$. Theorem 2 asserts that the set function $\langle E(\cdot) k(u), k(v)\rangle$ is absolutely continuous, whence the assertion of Theorem 3 follows.

CoROllaRy 1. The functions $\Phi(u ; \lambda)$ are for almost all $\lambda$ holomorphic functions of $u \in \Delta$ with the Maclaurin expansion (1.6). The mapping 
$U:\left\{f_{n}\right\} \rightarrow \sum_{0}^{\infty} f_{n} \phi_{n}$ is a unitary transformation of $l^{2}$ onto $L^{2}(d \rho)$ such that $U T U^{-1}=M$, where $M: g(\lambda) \rightarrow \lambda g(\lambda)$.

Proof. See the introduction.

4. Examples. The results obtained are:

EXAMPLE 1. $W(\theta)=\cos \theta$.

$$
\begin{array}{rlrl}
\rho^{\prime}(\lambda) & =\pi^{-1}\left(1-\lambda^{2}\right)^{1 / 2}, & |\lambda|<1 \\
& =0, & |\lambda| \geqq 1 . \\
\Phi(u ; \lambda) & =2^{1 / 2}\left(1-2 \lambda u+u^{2}\right)^{-1}, \\
\phi_{n}(\lambda) & =2^{1 / 2} C_{n}^{(1)}(\lambda), \text { where } C_{n}^{(1)}(\lambda)
\end{array}
$$

is the $n$th Gegenbauer polynomial of order 1 , cf. [2, p. 174].

Example 2. $W(\theta)=\sin \theta \cdot \rho^{\prime}(\lambda)$ is as in Example 1.

$$
\begin{aligned}
\Phi(u ; \lambda) & =2^{1 / 2}\left(1-2 i \lambda u-u^{2}\right)^{-1}, \\
\phi_{n}(\lambda) & =2^{1 / 2}(-i)^{n} C_{n}^{(1)}(\lambda) .
\end{aligned}
$$

Example 3. $W(\theta)=1$ if $|\theta| \leqq c ;=0$ otherwise. Here $0<c<\pi$.

$$
\rho^{\prime}(\lambda)=\frac{1}{\pi} \sin c \text { if } 0<\lambda<1,=0 \text { otherwise. }
$$

Set $\beta=-1 / 2 \pi \log \left(\lambda^{-1}-1\right)$. Then

$$
\begin{aligned}
\Phi(u ; \lambda) & =e^{c \beta}\left(1+e^{-2 \pi \beta}\right)^{1 / 2}\left(1-u e^{-i c}\right)^{-1 / 2-i \beta}\left(1-u e^{i c}\right)^{-1 / 2+i \beta}, \\
\phi_{n}(\lambda) & =e^{c \beta}\left(1+e^{-2 \pi \beta}\right)^{1 / 2} P_{n}^{(1 / 2)}(\beta, c),
\end{aligned}
$$

where $P_{n}^{(1 / 2)}(\beta, c)$ is the $n$th Pollaczek polynomial, cf. [5], of order $1 / 2$ for $(-\infty, \infty)$.

\section{REFERENCES}

1. A. Beurling, On two problems concerning linear transformations in Hilbert space, Acta Math. 81 (1949), 239-255.

2. A. Erdelyi et al., Higher transcendental functions, Vol. II, McGraw-Hill, New York, 1953.

3. P. Hartman and A. Wintner, On the spectra of Toeplitz's matrices, Amer. J. Math. 72 (1950), 359-366.

4. - The spectra of Toeplitz's matrices, Amer. J. Math. 76 (1954), 867-882.

5. F. Pollaczek, Sur une famille de polynômes orthogonaux qui contiente les polynomes d' Hermite et de Laguerre comme cas limites, C. R. Acad. Sci. Paris 230 (1950), 1563-1565.

6. M. Rosenblum, The absolute continuity of Toeplitz's matrices, Pacific J. Math. 10 (1960), 987-996.

The Institute for Advanced Study and UNIVERSITY OF VIRGINIA 